\title{
Recursos hídricos. Disponibilidad, variabilidad y gestión
}

\author{
Javier Lozano-Parra ${ }^{1}$
}

\begin{abstract}
El agua constituye un elemento de tal magnitud que está presente y juega un papel destacado en casi todos los procesos y ciclos naturales del planeta. Sin embargo, nuestra disponibilidad hídrica es limitada por naturaleza, es decir, aunque la Tierra contiene agua en abundancia, no siempre se encuentra en el momento y lugar adecuado y con la calidad apropiada. Por ejemplo, Chile es uno de los países con mayores recursos hídricos por persona del mundo. Sus precipitaciones alcanzaron un promedio de $761 \mathrm{~km}^{3}$ durante el periodo 1970-2000, de los cuales, alrededor de $250 \mathrm{~km}^{3}$ fueron devueltos a la atmósfera mediante el proceso de evapotranspiración. Esto significa que el territorio chileno fue capaz de generar un promedio hídrico superior a los $500 \mathrm{~km}^{3}$, susceptibles de ser utilizados cada año por el hombre. Sin embargo, el norte chileno presenta unos excedentes muy bajos en contraposición con el sur, más rico en recursos. Además, la disponibilidad varía cuando los recursos son considerados por espacios en función de su población. La abundancia del sur, escasamente poblado, contrasta con la insuficiencia de zonas con mayores densidades de población, como el centro y el norte, donde la actividad económica es mayor y la presión por los recursos más intensa.
\end{abstract}

Calcular de forma precisa la disponibilidad hídrica y sus variaciones espaciotemporales es esencial para controlar el suministro de alimentos, la producción energética, y la salud humana y ambiental. Sin embargo, se espera que en el futuro los recursos hídricos mundiales se vean sometidos a más presión. Por mencionar sólo algunos ejemplos, estudios recientes estiman que casi 5 mil millones de personas viven en zonas amenazadas por la disponibilidad hídrica, no obstante, en las próximas décadas se prevé un aumento exponencial de la población mundial. Asimismo, se estima que más del $80 \%$ de las aguas residuales globales no son tratadas, sin embargo, se espera que la población urbana mundial sobrepase ampliamente los 6 mil millones de personas el año 2050. Igualmente, la agricultura consume actualmente casi tres cuartas partes del agua utilizada en el planeta, no obstante, se pronostica un aumento del 70\% para 2050 en la demanda mundial de alimentos. Del mismo modo, casi dos tercios de los ecosistemas terrestres mundiales se encuentran amenazados, sin embargo, la precipitación y los caudales de los ríos que los sustentan son cada vez más variables debido al cambio climático. De esta forma, la escasez y la demanda por los recursos hídricos aumentan globalmente, lo que conlleva a una disparidad espacial que se ve reflejada en el aumento de las disputas por el agua. Algunos ejemplos pueden encontrarse en zonas del Creciente Fértil o del África subsahariana, donde el agua constituye uno de los principales factores que podrían explicar el surgimiento de conflictos armados.

La información fiable y objetiva sobre el estado de los recursos hídricos, así como de su utilización y gestión, constituye un elemento cada vez de mayor importancia para la toma de de- 
cisiones. En este contexto, los estudios enfocados en la componente hídrica se erigen como pieza fundamental para cubrir estas necesidades. Entender el ciclo hidrológico y su distribución espaciotemporal será un desafío crucial y clave del siglo XXI, tanto para la gestión eficaz de los recursos hídricos como para el mantenimiento de las sociedades humanas. Como un aporte en este sentido, el presente número ha agrupado cinco artículos que abordan esta temática: el uso de metodologías para evaluar el dinamismo de los recursos hídricos y las consecuencias de la disponibilidad de agua en el medio natural y antrópico.

El primer artículo es de Antonio Ceballos-Barbancho, José Manuel Llorente-Pinto, Esteban Alonso-González y Juan Ignacio López-Moreno, y tiene como objetivo mostrar la utilidad de la teledetección y modelos de balance energía para estudiar la estacionalidad y dinámica temporal del manto de nieve en una cuenca hidrográfica de tamaño pequeño (del río Tormes, España), ante la dificultad y coste que supone la instalación de dispositivos de medición en el campo.

En esta línea, Marcos Ruíz-Álvarez, Francisco Gomariz-Castillo y Francisco Alonso-Sarría proponen una metodología para realizar proyecciones espacio-temporales de la evapotranspiración de referencia (ETO) y el posible impacto del cambio climático en cuencas semiáridas. El estudio se desarrolla en la Demarcación Hidrográfica del Segura (España), utilizando para estimar la ETO el modelo de Hargreaves calibrado mensualmente mediante el método de Allen. Las proyecciones realizadas con todos los escenarios muestran un aumento significativo de la ETO durante el siglo XXI.

Continuando con las indagaciones metodológicas, Iván Vilchis-Mata, Carlos Félix Garrocho-Rangel y Carlos Díaz-Delgado modelan y simulan el "Ciclo Hidrosocial Urbano" para el periodo 2015-2030 de la Zona Metropolitana de Toluca, México. Los resultados permiten asegurar que en materia de disponibilidad de agua para 2030, la sostenibilidad de la ZMT está en riesgo. El balance hídrico del "ciclo hidrosocial urbano" y el patrón del abastecimiento de agua, registran un déficit en el balance oferta-demanda que se irá acentuando, dado el incremento continuo de la demanda y el declive de la oferta. Ante este escenario, resulta clave lograr eficiencia en la gobernanza y gobernabilidad del "ciclo hidrosocial urbano" de la ZMT, a través de una gestión integrada del agua como soporte de carácter científico-técnico para alimentar las políticas públicas.

En cuanto a los efectos que genera la disponibilidad de agua, el artículo de Javier Lozano-Parra, Carlos Lozano-Fondón, Manuel Pulido y Ramón García-Marín analiza las consecuencias de las variaciones meteorológicas sobre la dinámica del contenido de agua del suelo bajo diferentes estratos vegetales y la relación entre el contenido de agua del suelo y la producción de biomasa herbácea aérea durante el periodo de vida de las plantas anuales en un sector de la zona central de Chile. Los resultados muestran que entre el $57 \%$ y el $93 \%$ de la varianza en la dinámica vegetal puede ser explicada utilizando únicamente tres variables asociadas al ciclo hidrológico (precipitación, evapotranspiración y contenido hídrico del suelo) y, por tanto, un cambio en dicho ciclo podría amenazar el mantenimiento y productividad de estos ecosistemas.

Por su parte, Romina G. Sales y Bárbara Guida-Johnson reflexionan acerca de los desequilibrios territoriales que giran en torno a la presencia/ausencia del agua en las tierras secas de Mendoza, Argentina. Concluyen que debido a la dramática disminución del caudal del tramo inferior del río Tunuyán, los puesteros localizados aguas abajo del embalse Carrizal no tienen otra opción 
que acceder al agua subterránea para satisfacer sus necesidades, cuya calidad está relacionada a la salinidad de la misma y que si bien, el Estado está presente y busca solucionar este grave problema, aún falta por avanzar en que las políticas públicas consideren la mirada de los sujetos a la hora de diseñar las estrategias que permitan revertir los procesos de degradación ambiental.

Como es habitual en la revista, también se publican en este número trabajos que abordan otras temáticas afines. Tres de ellos, abordan interesantes problemáticas de las ciudades mexicanas; mientras que los otros dos artículos, se concentran en la injerencia de los pueblos indígenas en la gestión de sus territorios.

El texto de Fermín Alí Cruz Muñoz busca entender a partir de la construcción de un modelo, la lógica económico-territorial de la distribución del empleo en las zonas metropolitanas, específicamente, en la ciudad de México. Mediante el estudio estadístico y espacial se identifican la trascendencia y la magnitud de algunas variables que ayudan a explicar y predecir la organización de la actividad comercial de bienes de consumo inmediato.

Juan Hernández-Guerrero y Tamara Osorno-Sánchez analizan las diferencias ambientales de los paisajes urbanos de Juriquilla y Santa Rosa Jáuregui (al norte de la ciudad de Querétaro, México), y para ello elaboran un índice de calidad ambiental del paisaje urbano. Como resultado se logró identificar sitios prioritarios para mantenimiento y disposición de instrumentos de monitoreo ambiental, y se pudo observar que las desigualdades observadas a través de la estética urbana, infraestructura y servicios, son aristas del paisaje urbano que intervienen en la polarización y acentúan las diferencias ambientales.

El desarrollo inmobiliario asociado con la gentrificación extendida en la Ciudad de México es lo que discuten Patricia Olivera y Luis Salinas. Los autores señalan que la imposición de los proyectos genera exclusión y segregación urbana, desposesión de un bien patrimonial con un carácter fundamentalmente de uso y produce el desplazamiento hacia las periferias, fenómeno que pretende ser silenciado. Por lo tanto, afirman que solo las acciones articuladas de las organizaciones ciudadanas y del movimiento urbano popular podrán detener los procesos de una gentrificación extendida y excluyente en su ciudad.

El artículo de Francisco Molina Camacho, Jorge Constanzo Belmar y Cristián Inostroza Matus analiza desde la ecología política y la intercultural, el diálogo que se genera entre el pueblo lafkenche y las autoridades públicas en torno a los desastres naturales en la comuna de Saavedra (región de la Araucanía, Chile), tomando como contexto las implicancias socioambientales que trajo consigo el terremoto 9,5 richter y posterior tsunami ocurridos el año 1960. Los autores concluyen que gracias al reconocimiento que las autoridades públicas han tenido, respecto del conocimiento local del pueblo lafkenche en materia de desastres naturales se ha generado un diálogo intercultural que ha permitido la creación de un espacio de encuentro entre saberes, sentidos y prácticas, generando sinergias que han sido capaces de fortalecer los planes de emergencia comunales en materia de desastres naturales.

Finalmente, Hugo Romero-Toledo indaga sobre el fenómeno de la etnicidad aymara y la creación de etno-territorios en el marco de conflictos mineros y de la política indígena. Destaca que en el caso de los aymaras en el Norte Grande de Chile las poblaciones que persisten en el altipla- 
no y la precordillera han logrado, en alianza con la población que viven en las ciudades, levantar demandas sociales, culturales, ambientales y territorialidades, en dinámicas de negociación y conflicto con instituciones gubernamentales y con empresas mineras, que estabiliza ciertos etno-territorios como aymaras.

Como cierre de este "triple" número semitemático, se publican tres reseñas bibliográficas. La primera es un análisis crítico de Andrés Estefane al dossier especial № 69 de Revista de Geografía Norte Grande, en honor al profesor Dr. José Ignacio González. Por su parte, Nicole Jenne realiza una reseña del libro editado por Pedro lacobelli, Robert Cribb y Juan Luis Perello, "Asia y el Pacífico durante la Guerra Fría" (2018). En tanto, Andrés Bianchetti Saavedra critica el texto de Yasna Contreras Gatica, "Nuevos habitantes del centro de Santiago" (2016). 